\title{
Intelligent Design Automation for 2.5/3D Heterogeneous SoC Integration
}

\author{
Invited Paper \\ Iris Hui-Ru Jiang, Yao-Wen Chang, Jiun-Lang Huang, and Chung-Ping Chen \\ Department of Electrical Engineering and Graduate Institute of Electronics Engineering \\ National Taiwan University, Taipei 10617, Taiwan \\ Email: \{huirujiang, ywchang, jlhuang, cpchen\}@ntu.edu.tw
}

\begin{abstract}
As the design complexity grows dramatically in modern circuit designs, 2.5D/3D chip/package/board integration has become a key to beat process limitation for optimizing system performance and power consumption. Among the explored technologies, the wafer-level integrated fan-out (InFO) package-on-package (PoP) has been adopted by major companies such as TSMC to achieve high-density, high-performance, low-cost packaging solutions. To achieve a high-quality $2.5 \mathrm{D} / 3 \mathrm{D}$ heterogeneous integration system, we shall study the chip, package, and board codesign methodology with advanced packages and explore key techniques to handle the emerging challenges in physical design, timing, electrical effects, and testing. ${ }^{1}$
\end{abstract}

\section{KEYWORDS}

2.5D/3D heterogeneous integration, chip/package/board codesign, wafer-level integrated fan-out (InFO) package, package-on-package (PoP), physical design, timing, testing, system-level test, functionallike testing, electrical effects, routing, machine learning, Miller effect, nonlinear delay model, current source model

\section{INTRODUCTION}

As the design complexity grows dramatically in modern circuit designs, 2.5D/3D chip/package/board integration has become effective for optimizing system performance and power consumption. As a result, various $2.5 \mathrm{D} / 3 \mathrm{D}$ technologies have been explored, including stacked 3D ICs [16, 28], through-silicon-via-based (TSV-based) 3D ICs [32], interposer-based $2.5 \mathrm{D}$ packages $[5,15]$, and wafer-level chip-scale packages (WLCSPs) [35]. Among these technologies, WLCSPs have been adopted by major companies such as TSMC to achieve high-density, high-performance, low-cost packaging solutions.

\footnotetext{
${ }^{1}$ A preliminary version of this work is based on the proposal of Taiwan ERI special project funded by Taiwan MOST [1] under the grant no. 108-2218-E-002-074.

Permission to make digital or hard copies of all or part of this work for personal or classroom use is granted without fee provided that copies are not made or distributed for profit or commercial advantage and that copies bear this notice and the full citation on the first page. Copyrights for components of this work owned by others than ACM must be honored. Abstracting with credit is permitted. To copy otherwise, or republish, to post on servers or to redistribute to lists, requires prior specific permission and/or a fee. Request permissions from permissions@acm.org.

ICCAD '20, November 2-5, 2020, Virtual Event, USA

(C) 2020 Association for Computing Machinery.

ACM ISBN 978-1-4503-8026-3/20/11 \$\$15.00

https://doi.org/10.1145/3400302.3415767
}

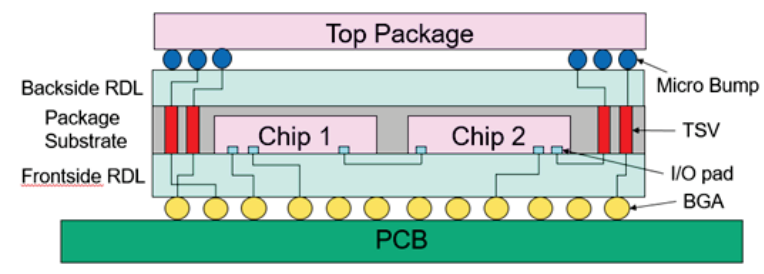

Figure 1: Cross-sectional view of InFO-PoP design.

In particular, the wafer-level integrated fan-out (InFO) packageon-package $(\mathrm{PoP})$ has become a popular advanced packaging technology, which usually consists of a bottom package with the InFO technique, and a top package stacked on the bottom package. Different from the traditional PoPs, there are frontside and backside re-distribution layers (RDLs) in the InFO PoP for signal redistributions. Figure 1 illustrates a cross-sectional view of an InFO-PoP design. Here, the through-silicon/InFO-vias (TSV/TIV) are used to provide vertical signal paths between the frontside and backside RDLs. Since there is no substrate and flip-chip bumps, the InFO$\mathrm{PoP}$ provides an average of $20 \%$ thinner height form factor than other PoP designs [38], e.g., the TSMC InFO-PoP has a thickness of only $0.8 \mathrm{~mm}$. In addition to the thickness reduction, InFO-PoP also outperforms other PoP technologies in thermal dissipation, reliability, electromigration, and compatibility of heterogeneous system integration [35].

To handle new design challenges, US DARPA has launched a fiveyear project called Electronic Resurgence Initiative (ERI) started from 2018. One main objective is the 3D Monolithic System-on-Chip realization, which aims at the manufacturing of 3D heterogeneous SoC integrated system via optical interconnect to realize high-speed computing and data accessing. A simple combination of traditional tools is not sufficient to achieve the desired design quality for chippackage-board integration of a heterogeneous system. Take physical design as an example, if the layout design between different levels are not considered simultaneously, the final layout could be only suboptimal in wirelength, timing, electrical performance, and testability.

Therefore, to achieve a high-quality $2.5 \mathrm{D} / 3 \mathrm{D}$ heterogeneous integration design, we shall study the chip, package, and board codesign methodology with advanced packages like InFO-PoPs considering some essential issues on physical design, timing, electrical effects, and testing to achieve globally optimized solutions. We elaborate these topics below: 


\subsection{Physical Design}

Existing placement and routing algorithms for 2.5D/3D heterogeneous integration design could lead to solution quality degradation under new design constraints and heterogeneous architectures, especially with InFO-PoPs. Therefore, a flexible, robust, and vertically integrated physical design flow for such heterogeneous integration designs is strongly desired; in particular, placement and routing technologies over the three physical levels, chips/packages/boards, of a complete heterogeneous system and their codesign need to be reinvented. We intend to start from the InFO technology for the package-level design and to develop novel cross-level co-optimization techniques, including (1) placement for components in the three levels, (2) RDL routing, and (3) board-level routing. For the placement problem, we propose to develop a chip-package co-placement algorithm to improve the wirelength and the routability in both chip-level and package-level designs. To further optimize the whole system, we propose to consider through-silicon-via placement for stacked PoPs and board-level placement. For the routing stage, we apply RDL routing and board-level routing to resolve routing congestion problems in the package and board levels. Three common design rule constraints during the routing stage should also be addressed: (1) the physical constraints, (2) the timing constraints, and (3) the electrical constraints.

\subsection{Timing analysis}

Existing academic timers for chip-level timing analysis, e.g., OpenSTA [3], OpenTimer [12], and iTimer [23], are developed based on nonlinear delay model (NLDM). The NLDM driver model is a voltage source with a constant resistance, where the output voltage is a linear function of time. The NLDM receiver model is a constant effective capacitance. For simplicity, NLDM only captures three output points. Nevertheless, capturing three points is insufficient to reflect the non-linearities behaviors of circuits at smaller geometries during static timing analysis (STA) because of (1) highly resistive interconnect, (2) nonlinear driver characteristics, (3) signal with long tail, and (4) unignorable Miller capacitance [31]. In contrast, the current source models (CSM) driver model is characterized by a current source with infinite driver resistance, where its output current is a nonlinear function of time and voltage. Thus, it provides better accuracy especially for high net impedance [2]. Specifically, CSM maintains accuracy by capturing the full output waveforms under varying slew and load conditions instead of a single delay and output slew value. However, waveforms require significant storage requirement. Therefore, we shall develop new STA approaches for achieving fast and accurate waveform propagation in chip-level analysis. Furthermore, there is not much research on chip-package co-analysis for timing of 2.5/3D heterogeneous integration, and we shall investigate new timing macro modeling and package level timing model for hierarchical STA.

\subsection{Electrical analysis}

Existing design methodology and flows to design chips and packages may not be effective with unexpected noise not only from the homogeneous monolithic layers, but also possibly the most complicated heterogeneous architectures. Furthermore, non-Manhattan routing in a $2.5 \mathrm{D}$ or even a $3 \mathrm{D}$ structure imposes even more difficulties to already hard magnetic coupling issues. Therefore, traditional electrical analysis and algorithms require immediate renovations and possibly renaissance. We propose to integrate the parasitics extraction, model reduction, and signal integrity analysis cross over the three levels of a complete heterogeneous non-rectilinear routing system.

\subsection{Testing}

Scan-based structural testing, while being the mainstream manufacturing testing methodology, is insufficient for today's highly integrated electronic systems. Due to their tight timing budget, these systems are more sensitive to process, voltage and temperature (PVT) variations and the context where they are used. As a result, structural testing that relies on automatic test equipment (ATE) to set and observe flip-flop values via scan chains fall short in the following aspects.

- During scan-based testing, the circuit under test (CUT) is operated in the test mode rather than the function mode. The discrepancy between the two modes may lead to overkill or test escape.

- Limited by its own timing accuracy, ATE is unable to reproduce arbitrary I/O timing conditions that may occur in reality. This is worsened by the test time and cost constraints.

To address the above issues, system-level test (SLT) becomes mandatory for digital systems, e.g., 2.5/3D SoCs, that demand high test quality.

Hence, our goal is to bridge the gap between the test and functional modes. Many techniques have been reported to generate functional-like test patterns by mimicking the switching activity characteristics $[34,36]$ or using reachable states as the scan-in flip-flop values [24, 29, 30, 33, 42]. However, neither approach is a comprehensive solution: switching activity is only part of the system behavior that affects test quality while reachability analysis is an NP-complete problem and cannot be scaled without significantly sacrificing the model accuracy.

We propose to develop a machine learning assisted pseudofunctional modeling technique that models the CUT to the extent necessary for fault detection. Compared to prior works, the constructed circuit model considers, in addition to switching activity, signal probabilities, association rules between signals, and other characteristics that impact test quality. Note that state reachability is implicitly modeled by signal association rules. To reduce the model construction efforts, close-loop machine learning strategies, e.g., reinforcement learning, will be employed. The constructed model is used to guide a pseudo-functional test generator. To accelerate the test generation process, search space and fault parallelisms will be explored.

\section{DESIGN AUTOMATION FOR HETEROGENEOUS INTEGRATION}

For aiming at the addressed big challenges, we intend to explore key techniques for the EDA flow and develop a complete tool chain to handle the emerging challenges with $2.5 \mathrm{D} / 3 \mathrm{D}$ heterogeneous system-on-chip (SoC) integration. Not only will point tools be developed to handle individual design processes, but also codesign 


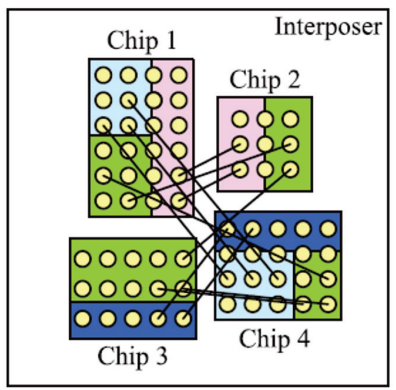

(a)

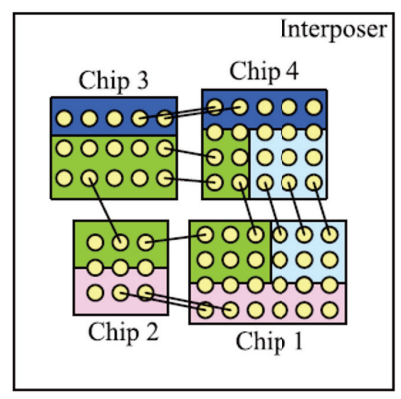

(b)
Figure 2: A routing-aware multiple chip planning scheme [13]. (a) An interposer floorplan without considering routability. (b) An interposer floorplan with considering routability.

methodology considering interactions of major design processes in different design levels will be addressed. We elaborate the addressed problems as follows.

\subsection{Physical Design}

With the rapidly growing demand for designs with high complexity, large I/O counts and small area, advanced packaging technologies such as the InFO technology has become more and more popular due to its high system performance, low leakage power, superior form factor, and strong capability of heterogeneous integration. However, the advanced packaging technologies bring new challenges in the physical design stage of the heterogeneous system integration flow. Existing placement and routing algorithms could lead to solution quality degradation under new design constraints and heterogeneous architectures. Therefore, a flexible, robust and vertically integrated physical design flow is strongly desired. To achieve a robust and flexible physical design flow for heterogeneous integration, we have different concerns in placement and routing. In each stage, there are two main issues to be considered: (1) heterogeneity and (2) integration. For heterogeneity, advanced technology nodes would incur new physical design rules and constraints, imposing different design requirements for chip-, package-, and board-level designs. Therefore, new placement and routing algorithms shall cope with issues arising from the heterogeneity of $2.5 \mathrm{D} / 3 \mathrm{D}$ system packaging systems, with good scalability. For integration, physical design must integrate algorithms for different specifications in both horizontal (heterogeneous dies or domains in a package or a board) and vertical (chip, package and board connection) aspects.

For the placement stage, chip-, package-, and board-level component planning is a key step in the physical design flow. Placement determines the physical location of each circuit component from the scale of nanometer to centimeter. Here, we mainly focus on one heterogeneous integration issue for placement: routing-aware placement for the vertical (chip-package-board) integration and cooptimization. As for the chip-package-board codesign, placement quality can strongly affect its routing result [13]. Figure 2(a) shows

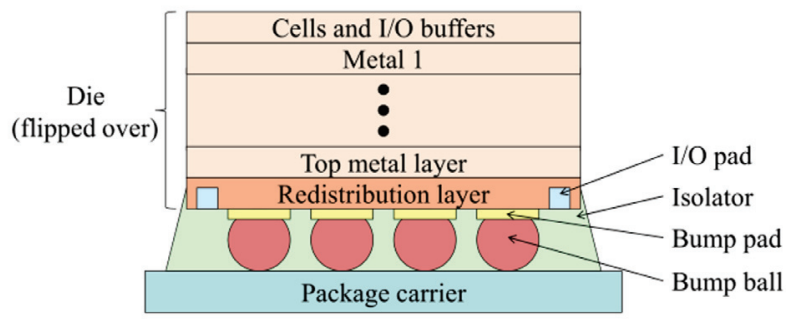

Figure 3: Cross view of a flip-chip [21].

a chip-package co-placement result without considering the interposer routability, while Figure 2(b) shows a co-placement result with better interposer routability. Routing congestion caused by an inappropriate placement result may cause routing failure for chips, packages, and/or boards. Therefore, it is better to consider chippackage-board co-placement when designing an overall physical design flow for heterogeneous integration.

For the routing stage, chip, package, board routing and advanced interconnect technologies have become the most complicated and easy-to-fail stage in the physical design flow. Routing determines the physical interconnections, including metal wires, vias, transmission mediums between chips and packages in a system. The task becomes even more challenging for heterogeneously integrated systems, which may contain chips with different technology nodes, packages with different domains, special analog design rules, nonuniform component sizes, pitches and spacing. For $2.5 \mathrm{D} / 3 \mathrm{D}$ system integration, it is desirable to consider heterogeneous routing structures in the package/board level for horizontal integration, chip-package-board routing optimization for vertical integration, and post-routing timing/electrical-driven optimization.

RDL routing provides signal redistribution in the chip level (in traditional flip-chip packages) [21] or in the package level (in interposers or package substrates). Existing RDL routing techniques can be classified by their targeting structures:

- RDL routing for a flip-chip package [7-11, 14, 25, 27, 39-41]: A flip-chip package would contain only one die. As shown in Figure 3, the RDL is attached on the top metal layer of a die and the router must complete the interconnections between I/O pad and bump balls. Figure 4 shows two common RDL structures.

- RDL routing for a 2.5D interposer-based package [15]: A 2.5D package would contain multiple chips. As shown in Figure 5, the RDL is on the silicon interposer and the router must complete two kinds of interconnection: (1) chip-to-chip connection and (2) chip-to-substrate/board connection.

- RDL routing for advanced packages [20, 26, 35]: Advanced packages may introduce a variety of structures. A router must consider these specific design structures and complete the required interconnections. See Figure 6 for a multiple chip RDL for an InFO package.

The aforementioned studies provide RDL routing techniques for flip-chip designs, and some of them consider the chip-package [8, 18, $19,22,37]$ or even chip-package-board codesign [13]. On the other hand, not much work discusses the RDL routing techniques for 


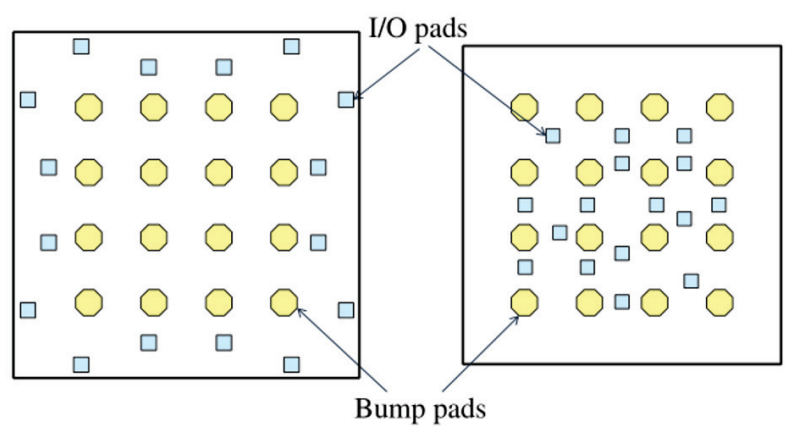

(a)

(b)

Figure 4: Two common RDL structures for flip-chip packages. (a) Peripheral-I/O flip-chip. (b) Area-I/O flip-chip [21].

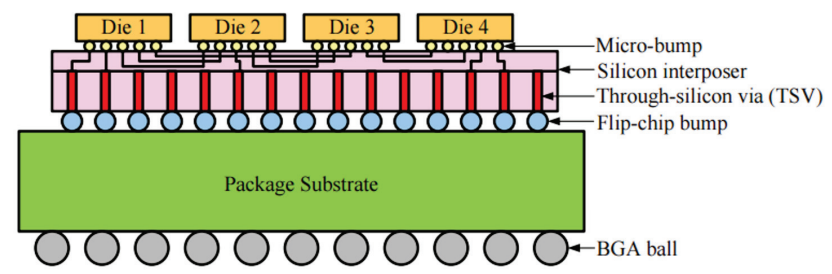

Figure 5: Cross view of a 2.5D IC Package [15].

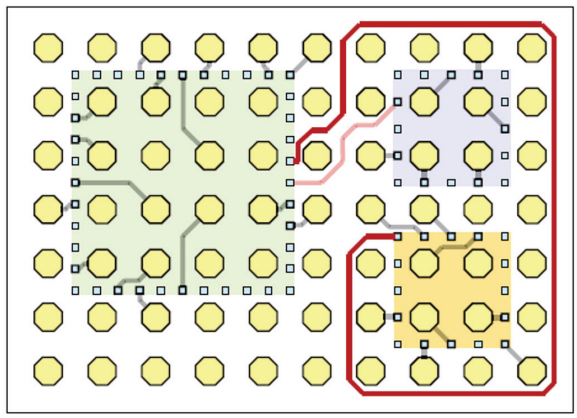

Figure 6: A multiple chip RDL routing scheme for an InFO package [26].

advanced packages [20,26], and even none considers the horizontal or vertical integration issues for advanced packages such as InFO packages.

For vertical integration of heterogeneous system, there would be heterogeneous components placed inside a single package, which may lead to different design constraints in different domains or routing regions. To our best knowledge, even modern commercial EDA tools cannot deal with this kind of integration problems well. Vertical integration is as important as the design of individual levels. Performing routing individually in chip, package, and board levels

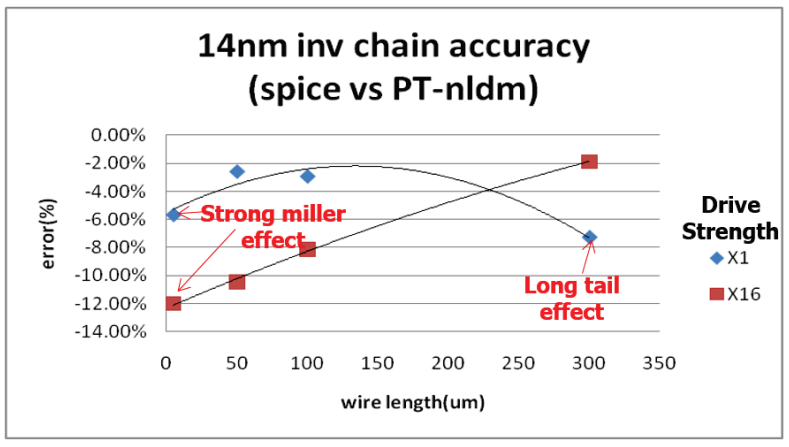

Figure 7: NLDM cannot capture nanometer timing [17].

without considering the vertical connections would cause solution quality degradation. In contrast, considering vertical integration often can achieve a much better solution. Therefore, we shall investigate the horizontal and vertical integration problems induced by advanced packages, and develop algorithms which can reduce the gap between heterogeneous components and different design levels.

\subsection{Timing Analysis}

Timing analysis is essential to achieve timing closure. Existing academic timers for chip-level analysis, e.g., OpenSTA [3], OpenTimer [12], and iTimer [23], are developed based on the nonlinear delay model (NLDM). The NLDM driver model is a voltage source with a constant resistance, which characterizes input-to-output delay and output slew with sensitivity to input slew, output load and side input states. The output voltage is a linear function of time. The NLDM receiver model is a constant effective capacitance. For simplicity, NLDM only captures three output points, e.g., $10 \%, 50 \%$, $90 \%$ of VDD. Nevertheless, capturing three points is insufficient to reflect non-linear behaviors of circuits at smaller geometries during STA because of highly resistive interconnect, nonlinear driver characteristics, signal with long tail, and unignorable Miller capacitance [31]. Figure 7 illustrates that the error generated by NLDM is ineligible with respect to SPICE simulation. In contrast, in current source models (CSM), the driver model is characterized by a current source with infinite driver resistance, output current is a nonlinear function of time and voltage. Thus, it provides better accuracy especially for high net impedance [2]. The CSM receiver model is characterized much like the NLDM receiver model with additional granularity to reflect sensitivities like miller capacitance, state of side inputs, input slew and output load. Specifically, CSM maintains accuracy by capturing the full output waveforms under varying slew and load conditions instead of a single delay and output slew value. Fast and accurate effective capacitance calculation and waveform generation are the key to CSM delay and slew computation. Moreover, a simple rule of thumb is that the file size of a new CSM is about 10X larger than that of NLDM. Therefore, new STA approaches are desired to deliver fast and accurate waveform propagation in chip-level analysis. 


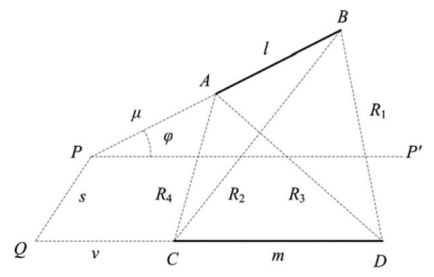

(a)

$$
\begin{aligned}
M= & \frac{\mu_{0} \cos \varphi}{2 \pi}\left[-\mu \tanh ^{-1} \frac{m}{R_{3}+R_{4}}-\nu \tanh ^{-1} \frac{l}{R_{2}+R_{3}}+(\mu+l) \tanh ^{-1} \frac{m}{R_{1}+R_{2}}\right. \\
& \left.+(\nu+m) \tanh ^{-1} \frac{l}{R_{1}+R_{4}}-\frac{\Omega s}{2 \sin \varphi}\right], \\
\Omega= & \tan ^{-1} \frac{s^{2} \cos \varphi+(\mu+l)(\nu+m) \sin ^{2} \varphi}{s R_{1} \sin \varphi}-\tan ^{-1} \frac{s^{2} \cos \varphi+(\mu+l) \nu \sin ^{2} \varphi}{s R_{2} \sin \varphi} \\
& +\tan ^{-1} \frac{s^{2} \cos \varphi+\mu \nu \sin ^{2} \varphi}{s R_{3} \sin \varphi}-\tan ^{-1} \frac{s^{2} \cos \varphi+\mu(\nu+m) \sin ^{2} \varphi}{s R_{4} \sin \varphi} .
\end{aligned}
$$

Figure 8: Inductance formulae for non-rectilinear geometry.

\subsection{Electrical Analysis}

With the rapidly growing demand for designs with high complexity, large I/O counts but small area, the system-in-package ( $\mathrm{SiP}$ ) has become a practical and promising solution for integrating complicated systems. However, the advanced packaging technologies bring new challenges in the coupling between the packages and die integration of the heterogeneous system integration. The existing design methodology and flow to design die and packaging may not be effective with unexpected signal integrity issues not only from the monolithic layers, but also from the most complicated heterogeneous architectures especially in the non-rectilinear structure. Therefore, a cross-layer, cross material, integrated, parasitic extraction and comprehensive signal integrity analysis, and simulation methodology are of great importance.

The two TSMC new packaging technologies, InFO and CoWoS (Chip on Wafer on Substrate), are recently developed to minimize the packaging sizes and power consumption. However, the compact sizes of these I/O bumps and chips and non-rectilinear geometries which impose many signal integrity issues for long range effects. For example, not only the coupling capacitance, but also the coupling inductance from I/Os and even dies may induce huge noises to full chips. With a long antenna laying across the working chip, a number of nets will be influenced by this big antenna. Besides, the return paths issues for inductors and reductions are quite difficult for non-Manhattan geometry. Furthermore, to speed up the packingdie co-analysis, there is a need to perform model reduction to the already extracted RLCS models. The stability and passivity of model order reduction are to ensure the accuracy and reduce the analysis turnaround time.

The packaging chip co-analysis issues to be investigated include the parasitic extraction for non-Manhattan geometry, packagingchip signal integrity co-analysis, and model reduction from the chip level, the package level, to the board level, which covers the entire homogeneous and heterogeneous system. This integration may look straightforward. However, it engages a major change across different horizons in the design of chips, packages, and boards, and may bring a fundamental change to the ICs and level up to the system design flows.

\section{$2.4 \quad$ Testing}

One of our goals is to bridge the gap between the scan-testing mode and the functional mode. In this way, CUT can be tested in a more functional-like manner, which helps improve test quality and reduce test yield loss. To reach this goal, we will develop a machine learning assisted pseudo-functional test pattern generator (ML-PFTPG). Figure 9 depicts the proposed ML-PFTPG of which the two main components are (1) ML-assisted pseudo-functional modeling, and (2) the pseudo-functional test generator.

Past efforts to model the CUT can be categorized as follows.

- Characteristics based modeling. The goal is to match CUT's behavior in the interested characteristics. For example, [34, 36] tune test patterns so that the resulting IR-drop profiles are close to those in the functional mode. One challenge of these approaches is to define the target profile(s).

- Reachability modeling. The idea is to ensure that the scan-in states are reachable or close to reachable states [24, 29, 30, 33, 42]. Since reachability analysis is an NP-complete problem, one has to trade model accuracy for computation complexity.

Compared to previous efforts to construct pseudo-functional models, the ML-assisted pseudo-functional modeling differs in the following aspects.

- A closed loop learning strategy, e.g., reinforcement learning, will be adopted. This improves sample efficiency, i.e., more informative simulation traces, and reduces the model construction efforts by concentrating on the currently targeted faults.

- In addition to state reachability and signal switching activity, circuit characteristics such as signal probabilities and the association rules between signals, will also be considered. By mimicking more functional characteristics, the accuracy of reachability analysis can be relieved.

For conventional test pattern generators, fault coverage and pattern counts are the main concerns. However, to further improve test quality and reduce test cost, recent test pattern generators also take into account constraints such as power consumption, compatibility with test compression schemes, and even diagnosability. Similarly, the proposed pseudo-functional test generator will be guided by the constructed pseudo-functional CUT model, which will inevitably incur CPU overhead. To confine the CPU times, we will introduce parallelism in the following two aspects.

- Search space parallelism. The additional constraints, i.e., the CUT's pseudo-functional model, tend to reduce the solution space. Thus, search space parallelism, which is known to be effective for hard-to-detect faults, will be introduced.

- Fault parallelism. For further CPU time reduction, fault parallelism will be introduced to dispatch un-detected faults to available test generation threads.

Preliminary results in $[4,6]$ demonstrate the feasibility of generating functional-like test patterns and RL-based test pattern generation, respectively. 


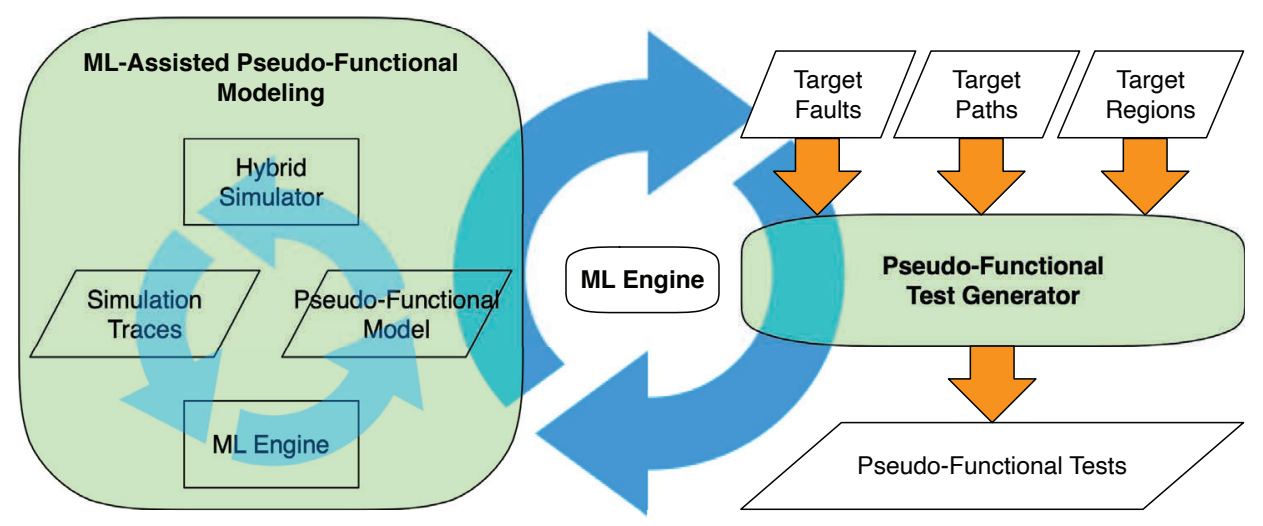

Figure 9: ML-assisted pseudo-functional test pattern generator.

\section{FUTURE RESEARCH DIRECTIONS}

Here we provide some future research topics that need to be addressed to advance the $2.5 \mathrm{D} / 3 \mathrm{D}$ heterogeneous system integration.

- Routing for heterogeneous chip/package/board designs: It is desirable to develop a package-level RDL router with heterogeneous components and routing region constraints for multiple-chip packages (say, InFO packages), and a vertical routing integrated router with chip-package-board routing codesign for advanced system integration (say, InFO PoPs).

- Placement-and-routing for chip-package-board integration: It is desirable to develop a chip-package-board placer and router with heterogeneous packages (including InFOs, flipchips, and 2.5D/3D integration), and a physical/timing/electricalaware chip/package/board router (say, a router with differential pairs).

- Irregular I/O structures: Flip-chips with regular or staggered I/O structures are typical in traditional package designs; however, irregular I/O structures are commonly seen in modern $2.5 \mathrm{D} / 3 \mathrm{D}$ heterogeneous integration to address the different design rules and requirements from various components. Such irregular I/O structures would require new tile models to capture the routing resource utilization and more advanced routing algorithms to handle layout heterogeneity.

- Routing with multiple design rules: Routing with uniform design rules are typical in traditional designs; however, routes with multiple wire widths and spacing are often seen in modern $2.5 \mathrm{D} / 3 \mathrm{D}$ heterogeneous integration to address various requirements from heterogeneous components. Such non-uniform rules would require new models to capture the irregular via and non-uniform wiring structures and advanced routing algorithms with efficient geometry query capability to handle the non-uniform routing structure.

- Optical Routing: Semiconductor giants like Intel and TSMC are actively exploring the technologies for integrating electrical and optical interconnections in a modern $2.5 \mathrm{D} / 3 \mathrm{D}$ heterogeneous integration system. Compared with electrical routes (metal wires), the optical routes (waveguides) can provide higher bandwidth, lower power consumption, and better routability, but require larger area per net and additional electrical-optical conversion components. When applying the optical interconnect technology to package/board level routing, the optical and electrical route codesign issue becomes important.

- CSM timing macro modeling: Existing timing macro models reduce the timing graph size by applying graph reduction techniques, e.g., serial and parallel merging, biclique-star replacement, tree merging. However, most of existing reduction techniques do not consider regularity of subdesign. For $2.5 / 3 \mathrm{D}$ heterogeneous integration, we shall investigate new timing macro modeling based on CSM and with emphasis on regularity extraction. Moreover, with these new timing macro models and package-level timing models, we can perform hierarchical analysis to achieve chip-package co-analysis.

- AI-assisted electrical analysis: To expedite the system-level electrical analysis with InFO packages, widely available AI techniques could be adopted to capture non-rectilinear characteristics in a short time.

- Re-producing real-life inter-die timing conditions: While we address the discrepancy between the scan-test mode and the functional mode by testing each die in a $2.5 / 3 \mathrm{D}$ SoC using more functional-like test patterns, the problem of reproducing real-life inter-die timing conditions is left unanswered and will be investigated in the future.

\section{CONCLUSION}

2.5D/3D chip/package/board integration has become a key to beat process limitation for further advancement. To achieve a highquality $2.5 \mathrm{D} / 3 \mathrm{D}$ heterogeneous integration system, in this paper, we study the chip, package, and board codesign methodology with advanced packages and explore key techniques to handle the emerging challenges in physical design, timing, electrical effects, and testing. There are still many opportunities for future research to advance $2.5 \mathrm{D} / 3 \mathrm{D}$ heterogeneous SoC integration.

\section{REFERENCES}

[1] Y.-W. Chang, I. H.-R. Jiang, C.-P. Chen, and J.-L. Huang, "Intelligent Design Automation for 2.5/3D Heterogeneous SoC Integration," Proposal of Taiwan ERI Special Project, 2019. 
[2] J. Bhasker and R. Chadha, "Static Timing Analysis for Nanometer Designs: A Practical Approach," Springer, 2009.

[3] J. Cherry, "OpenSTA Engine," 2019. https://github.com/The-OpenROAD-Project/ OpenSTA.

[4] C.-Y. Chen, C.-H. Cheng, J.-L. Huang, and K. Chakrabarty, "Functional-Like Transition Delay Fault Test-Pattern Generation using a Bayesian-Based Circuit Model," in Proceedings of the European Test Symposium, pp. 1-6, Talinn, Estonia, May 2020.

[5] A. Coskun, F. Eris, A. Joshi, A. B. Kahng, Y. Ma, and V. Srinivas, "A Cross-Layer Methodology for Design and Optimization of Networks in 2.5D Systems," in Proceedings of International Conference on Computer-Aided Design, p. 101, San Jose, CA, November 2018.

[6] C.-Y. Chen and J.-L. Huang, "Reinforcement-Learning Based Test Program Gener ation for Software-Based Self-Test," in Proceedings of the Asian Test Symposium, pp. 73-78, Kolkata, India, December 2019.

[7] J.-W. Fang and Y.-W. Chang, "Area-I/O Flip-Chip Routing for Chip-Package CoDesign Considering Signal Skews," IEEE Transactions on Computer-Aided Design of Integrated Circuits and Systems, Vol. 29, No. 5, pp. 711-721, May 2010.

[8] J.-W. Fang, K.-H. Ho, and Y.-W. Chang, "Routing for Chip-Package-Board CoDesign Considering Differential Pairs," in Proceedings of International Conference on Computer-Aided Design, pp. 512-517, San Jose, CA, November 2008.

[9] J.-W. Fang, C.-H. Hsu, and Y.-W. Chang, "An Integer-Linear-Programming-Based Routing Algorithm for Flip-Chip Designs," IEEE Transactions on Computer-Aided Design of Integrated Circuits and Systems, Vol. 28, No. 1, pp. 98-110, January 2009.

[10] J.-W. Fang, I.-J. Lin, Y.-W. Chang, and J.-H. Wang, "A Network-Flow-Based RDL Routing Algorithm for Flip-Chip Design," IEEE Transactions on Computer-Aided Design of Integrated Circuits and Systems, Vol. 26, No. 8, pp. 1417-1429, August 2007

[11] J.-W. Fang, M. D. F. Wong, and Y.-W. Chang, "Flip-Chip Routing with Unified AreaI/O Pad Assignment for Package-Board Co-Design," in Proceedings of Design Automation Conference, pp. 336-339, San Francisco, CA, July 2009.

[12] T.-W. Huang, "OpenTimer: a High-performance Timing Analysis Tool for VLSI Systems," 2019. https://github.com/OpenTimer.

[13] Y.-K. Ho and Y.-W. Chang, "Multiple Chip Planning for Chip-Interposer Codesign," in Proceedings of Design Automation Conference, pp. 27-32, Austin, TX, June 2013

[14] Y.-K. Ho, H.-C. Lee, P.-W. Lee, Y.-W. Chang, C.-F. Chang, I.-J. Lin, and C.-F. Shen, "Obstacle-Avoiding Free-Assignment Routing for Flip-Chip Designs," IEEE Transactions on Computer-Aided Design of Integrated Circuits and Systems, Vol 33, No. 2, pp. 224-236, February 2014

[15] Y.-K. Ho and Y.-W. Chang, "Multiple Chip Planning for Chip-Interposer Codesign," in Proceedings of Design Automation Conference, p. 27, Austin Texas, May 2013.

[16] D. H. Kim, K. Athikulwongse, and S. K. Lim, "A Study of Through-Silicon-Via Impact on the 3D Stacked IC Layout," in Proceedings of International Conference on Computer-Aided Design, 2009.

[17] M.-S. Kim, S. Heo, D. Lee, D. Hyun, B. S. Kim, B. Lee, C. Rim, H. Won, K. Kim, "Timing Analysis with Waveform Propagation," Design Automation Conference User Track, 2013

[18] M.-F. Lai and H.-M. Chen, "An Implementation of Performance-Driven Block and I/O Placement for Chip-Package Codesign," in Proceedings of International Symposium on Quality Electronic Design, pp. 604-607, San Jose, CA, March 2008

[19] R.-J. Lee and H.-M. Chen, "Row-Based Area-Array I/O Design Planning in Con current Chip-Package Design Flow," in Proceedings of Asia South Pacific Design Automation Conference, pp. 837-842, Yokohama, Japan, January 2011.

[20] T.-C. Lin, C.-C. Chi, and Y.-W. Chang, "Redistribution Layer Routing for WaferLevel Integrated Fan-Out Package-on-Packages," in Proceedings of International Conference on Computer-Aided Design, pp. 561-568, Irvine, CA, November 2017.

[21] H.-C. Lee, Y.-W. Chang, and P.-W. Lee, "Recent Research Development in FlipChip Routing," in Proceedings of International Conference on Computer-Aided Design, pp. 404-410, San Jose, CA, November 2010.

[22] K.-S. Lin, H.-W. Hsu, R.-J. Lee, and H.-M. Chen, "Area-I/O RDL Routing for ChipPackage Codesign Considering Regional Assignment," in Proceedings of IEEE Electrical Design of Advanced Package Systems Symposium, pp. 1-4, Singapore, Singapore, December 2010

[23] P.-Y. Lee, I. H.-R. Jiang, C.-R. Li, W.-L. Chiu, and Y.-M. Yang, "iTimerC 2.0: Fast Incremental Timing and CPPR Analysis," in Proceedings of International Conference on Computer-Aided Design, pp. 890-894, Austin, TX, November 2015.

[24] Y.-C. Lin, F. Lu, K. Yang, and K.-T. Cheng, "Constraint Extraction for PseudoFunctional Scan-based Delay Testing," in Proceedings of Asia and South Pacific Design Automation Conference, pp. 166-171, Shanghai, China, July 2005

25] C.-W. Lin, P.-W. Lee, Y.-W. Chang, C.-F. Shen, and W.-C. Tseng, "An Efficient Pre-Assignment Routing Algorithm for Flip-Chip Designs," IEEE Transactions on Computer-Aided Design of Integrated Circuits and Systems, Vol. 31, No. 6, pp 878-889, June 2012.

[26] B.-O. Lin, T.-C. Lin, and Y.-W. Chang, "Redistribution Layer Routing for Integrated Fan-Out Wafer-Level Chip-Scale Packages," in Proceedings of International Conference on Computer-Aided Design, pp. 1-8, Austin, TX, November 2016.
[27] X. Liu, Y. Zhang, G. K. Yeap, C. Chu, J. Sun, and X. Zeng, "Global Routing and Track Assignment for Flip-Chip Designs," in Proceedings of Design Automation Conference, pp. 90-93, Anaheim, CA, June 2010.

[28] X.-D. Liu, Y.-F. Zhang, G. Yeap, and X. Zeng, "An Integrated Algorithm for 3D-IC TSV Assignment," in Proceedings of Design Automation Conference, pp. 652-657, San Diego, CA, June 2011.

[29] E. K. Moghaddam, J. Rajski, M. Kassab, and S. M. Reddy, "At-Speed Scan Test with Low Switching Activity," in Proceedings of VLSI Test Symposium, pp. 177-182, Santa Cruz, CA, April 2010.

[30] I. Pomeranz, "On the Generation of Scan-based Test Sets with Reachable States for Testing under Functional Operation Conditions," in Proceedings of Design Automation Conference, pp. 928-933, San Diego, CA, July 2004.

[31] V. Shah and D. Patel, "Distorted Waveform Phenomena in $7 \mathrm{~nm}$ Technology Node and its Impact on Signoff Timing Analysis," Design and Reuse, 2019. https://www.design-reuse.com/articles/46076/ distorted-waveform-phenomena-7nm-signoff-timing-analysis.html.

[32] M. Sunohara, T. Tokunaga, T. Kurihara, and M. Higashi, "Silicon Interposer with TSVs (Through Silicon Vias) and Fine Multilayer Wiring," in Proceedings of Electronic Components and Technology Conference, pp. 847-852, Lake Buena Vista, FL, June 2008.

[33] A. Touati, A. Bosio, L. Dilillo, P. Girard, A. Virazel, P. Bernardi, and M. S. Reorda, "Exploring the Impact of Functional Test Programs Re-used for Power-Aware Testing," in Proceedings of Design, Automation and Test in Europe, pp. 1277-1280, Grenoble, France, March 2015.

[34] L.-C. Tsai and J.-Z. Li, J.-L. Huang, A. Shih, and Z. F. Conroy, "An IR-Drop Guided Test Pattern Generation Technique," In Proceedings of International Symposium on VLSI Design, Automation and Test, pp. 1-4, Hsinchu, Taiwan, June 2016.

[35] C.-F. Tseng, C.-S. Liu, C.-H. Wu and D. Yu, "InFO (Wafer Level Integrated FanOut) Technology," in Proceedings of Electronic Components and Technology Conference, pp. 1-6, Las Vegas, NV, June 2016.

[36] X. Wen, Y. Nishida, K. Miyase, S. Kajihara, P. Girard, M. Tehranipoor, and L.-T. Wang, "On Pinpoint Capture Power Management in At-Speed Scan Test Generation," in Proceedings of International Test Conference, pp. 1-10, Anaheim, CA, November 2012.

[37] J. Xiong, Y.-C. Wong, E. Sarto, and L. He, "Constraint Driven I/O Planning and Placement for Chip-Package Co-Design," in Proceedings of Asia South Pacific Design Automation Conference, pp. 207-212, Yokohama, Japan, January 2006.

[38] D. Yu, "A New Integration Technology Platform: Integrated Fan-out Waferlevel-packaging for Mobile Applications," in Proceedings of Symposium on VLSI Technology, pp. T46-T47, June 2016.

[39] J.-T. Yan and Z.-W. Chen, "IO connection Assignment and RDL Routing for Flip-Chip designs," in Proceedings of Asia South Pacific Design Automation Conference, pp. 588-593, Yokohama, Japan, January 2009.

[40] J.-T. Yan and Z.-W. Chen, "Pre-Assignment RDL Routing via Extraction of Maximal Net Sequence," in Proceedings of IEEE International Conference on Computer Design, pp. 65-70, Amherst, MA, October 2011.

[41] T. Yan and M. D. F. Wong, "A Correct Network Flow Model for Escape Routing," in Proceedings of Design Automation Conference, pp. 332-335, San Francisco, CA, July 2009.

[42] Z. Zhang, S. M. Reddy, and I. Pomeranz, "On Generating Pseudo-Functional Delay Fault Tests for Scan Designs," in Proceedings of International Symposium on Defect and Fault Tolerance in VLSI Systems, pp. 398-405, Monterey, CA, October 2005 . 